\title{
EXISTENCE-AND-UNIQUENESS AND MEAN-SQUARE BOUNDEDNESS OF THE SOLUTION TO STOCHASTIC CONTROL SYSTEMS ${ }^{\dagger}$
}

\author{
PEILIN LU AND CAIXIA GAO*
}

\begin{abstract}
This paper mainly deals with the stochastic control system, the existence and uniqueness of solutions and the behavior of solutions are investigated. Firstly, we obtain sufficient conditions which guarantee the existence and uniqueness of solutions to the stochastic control system. And then, boundedness of the solution to the system is achieved under mean-square linear growth condition.
\end{abstract}

AMS Mathematics Subject Classification : 93E03, 34H05.

Key words and phrases : stochastic control systems, existence and uniqueness, mean-square boundedness.

\section{Introduction}

Stochastic control theory is one of the most important part of control theory and stochastic optimal theory, due to their wide applications in engineering, life science and finance, see $[1,2]$. For the following stochastic differential equation

$$
\left\{\begin{array}{l}
d x(t)=f(t, x(t)) d t+g(t, x(t)) d B(t) \quad t \in\left[t_{0}, T\right] \\
x(t)=x_{0}
\end{array}\right.
$$

Where $x(t) \in R^{n}, f:[0, T] \times R^{n} \rightarrow R^{n}, g:[0, T] \times R^{n} \rightarrow R^{n \times m}$ are determined function with respect to $t$ and $x$. The existence and uniqueness of solutions and the behavior of solutions were investigated in [3], which showed that if for any $(t, x) \in[0, T] \times R^{n}$,

$$
|f(t, x)|+|g(t, x)| \leq C(1+|x|)
$$

Received October 20, 2012. Revised November 21, 2012. Accepted November 28, 2012. ${ }^{*}$ Corresponding author. ${ }^{\dagger}$ This work was supported by the Natural Science Foundation of China (Grant No. 11261033).

(c) 2013 Korean SIGCAM and KSCAM. 
and for any $x, y \in R^{n}$ and $t \in[0, T]$,

$$
|f(t, x)-f(t, y)|+|g(t, x)-g(t, y)| \leq K|x-y|, \quad K \geq 0
$$

then there exists a unique $t$-continuous solution $x(t)$, which is adapted to a filtration $F_{t}^{x_{0}}$ generated by $x_{0}$ and $B(s), s \leq t$, and

$$
E \int_{0}^{T}|x(t)|^{2} d t<\infty
$$

Also, Hu Guixin, in [4], gave the proof of the existence and uniqueness theorem for stochastic differential equations with random coefficients as follows.

$$
d x(t)=f(\omega, t, x(t)) d t+g(\omega, t, x(t)) d B(t)
$$

In this paper, we consider the existence and uniqueness of solutions to the following stochastic control system:

$$
\left\{\begin{array}{l}
d x^{u}(t)=f(t, x(t), u(t, x(t))) d t+g(t, x(t), u(t, x(t))) d B(t) \quad t \in[0, T] \\
u(t, x(t)) \in U
\end{array}\right.
$$

Where $u(t, x(t)) \in U$ is a Markov control function which depend only on $t$ and on the state $x(t)$ at the instant $t$.

The organization of this paper is as follows. Firstly, we give some preliminary concepts and definitions in Section 2. In Section 3, we investigate the existence and uniqueness of solutions and behavior of solutions to the stochastic control system. And we give the conclusion at the last section.

\section{Preliminary concepts and definitions}

In this section, we introduce some basic concepts and Lemmas which are used throughout this paper.

Let $X$ and $X_{n}$, where $n \geq 1$, denote $R^{n}$-valued random variables defined on a probability space $(\Omega, \mathcal{F}, P)$. The following two convergence concepts can be found application in probability theory, see [5].

(a) If there exists a set of measure zero $N \in \mathcal{F}$ such that, for all $\omega \notin N$, the sequence $X_{n}(\omega) \in R^{n}$ converges in the usual sense to $X(\omega) \in R^{n}$, then $\left\{X_{n}\right\}$ is said to converge almost certainly $[P]$ or with probability 1 to $X$. we write

$$
a c-\lim _{n \rightarrow \infty} X_{n}=X \text {. }
$$

(b) If for every $\varepsilon>0$,

$$
P_{n}(\varepsilon)=P\left\{\omega:\left|X_{n}(\omega)-X(\omega)\right|>\varepsilon\right\} \rightarrow 0 \quad(n \rightarrow \infty),
$$

then $\left\{X_{n}\right\}$ is said to converge stochastically or in probability to $X$, and we write

$$
s t-\lim _{n \rightarrow \infty} X_{n}=X .
$$

Lemma 2.1 (Chebyshev Inequality). If $c>0, p>0, X \in L^{p}$, then

$$
P\{\omega:|X(\omega)| \geq c\} \leq c^{-p} E|X|^{p}
$$


Lemma 2.2 (Borel-Cantelli Lemma). If $\left\{A_{k}\right\} \subset F$ and $\sum_{k=1}^{\infty} P\left(A_{k}\right)<\infty$, then

$$
P\left(\limsup _{k \rightarrow \infty} A_{k}\right)=0
$$

Lemma 2.3. Let $G, G_{1}, G_{2}, \ldots, G_{n}$ is $n \times m$ matrix valued function on $M_{2}\left[t_{0}, t\right]$ and $B(t)$ is m-dimensional Brownian motion. If

$$
\lim _{n \rightarrow \infty} \int_{t_{0}}^{t} E\left|G_{n}(s)-G(s)\right|^{2} d s=0
$$

then

$$
s t-\lim _{n \rightarrow \infty} \int_{t_{0}}^{t} G_{n} d B(t)=\int_{t_{0}}^{t} G d B(t)
$$

Lemma 2.4 (Gronwall Inequality). Suppose $x(t), L(t) \in C([a, b] ; R)$, constant $c \geq 0$, such that

$$
0 \leq x(t) \leq c+\int_{a}^{t} L(s) x(s) d s
$$

then for any $t \in[a, b]$, we have

$$
0 \leq x(t) \leq c e^{\int_{a}^{t} L(s) d s}
$$

In particular, when $c=0, x(t)=0$.

\section{The Solution of Stochastic Control Systems}

Let $(\Omega, \mathcal{F}, P)$ be a probability space with filtration $\left\{\mathcal{F}_{t}\right\}$ and $(B(t))_{t \geq 0}$ be a $R^{n}$-valued standard Brownian motion. We assume $\mathcal{F}_{t}=\sigma\{(B(s)), 0 \leq s \leq$ $t\}$ and consider the following $n$-dimensional stochastic control system:

$$
\left\{\begin{array}{l}
d x^{u}(t)=f(t, x(t), u(t, x(t))) d t+g(t, x(t), u(t, x(t))) d B(t) \quad t \in[0, T] \\
u(t, x(t)) \in U
\end{array}\right.
$$

Where $x^{u}(t) \in R^{n}, f:[0, T] \times R^{n} \times U \rightarrow R^{n}, g:[0, T] \times R^{n} \times U \rightarrow R^{n \times m}$ and $B(t)$ is $m$-dimensional Brownian motion. The functions $u(t, x(t))$ is a control function whose value we can choose in the given Borel set $U \subset R^{p}$ of admissible control functions. we shall confine ourselves here to Markov control functions which depend only on $t$ and on the state $x(t)$ at the instant $t$ and not on the value of $x(s)$ for $(s<t)$.

Let $g(t, x(t), u(t, x(t)))$ be a $m \times n$ matrix valued function, $f(t, x(t), u(t, x(t)))$ be a $n$-vector valued function and $u(t, x(t))$ be a $p$-vector valued function, and

(i) For each $u, g(t, x(t), u(t, x(t)))$ and $f(t, x(t), u(t, x(t)))$ are continuous in $(t, x)$;

(ii) the functions $g(t, x(t), u(t, x(t)))$ and $f(t, x(t), u(t, x(t)))$ are measurable with respect to $t, x$ and $u$, for $t \in[0, T], x \in R^{n}$ and $u \in R^{p}$ with $T>0$;

(iii) for any $(t, x) \in[0, T] \times R^{n}, g(t, x(t), u(t, x(t))), f(t, x(t), u(t, x(t))) \in$ $L^{2}(P)$; for any $x \in R^{n}$, such that $g(t, x(t), u(t, x(t)))$ and $f(t, x(t), u(t, x(t)))$ are $\mathcal{F}_{t}$-adapted; 
(iv) there exist constants $K$ and $C$, for all $t \in[0, T]$, and $x, y \in R^{n}$, such that (a) (mean square linear growth condition)

$$
\begin{aligned}
& E|f(t, x(t), u(t, x(t)))|^{2}+E|g(t, x(t), u(t, x(t)))|^{2} \\
\leq & C\left(1+|x(t)|^{2}+|u(t, x(t))|^{2}\right)
\end{aligned}
$$

(b) (mean square Lipschitz condition)

$$
\begin{gathered}
E|f(t, x(t), u(t, x(t)))-f(t, y(t), u(t, y(t)))|^{2} \\
\quad+E|g(t, x(t), u(t, x(t)))-g(t, y(t), u(t, y(t)))|^{2} \\
\left.\leq\left. K(\mid x(t)-y(t))\right|^{2}+|u(t, x(t))-u(t, y(t))|^{2}\right)
\end{gathered}
$$

(v) control set $U$ is a bounded set, that is, for any $u \in U$, there exists constant $M>0$, such that

$$
\|u\| \leq M
$$

(vi) there exists constant $L$, for any $u \in U$ :

$$
E \mid u(t, x(t))-u(t, y(t)))\left.\right|^{2} \leq\left. L(\mid x(t)-y(t))\right|^{2}
$$

Definition 3.1. $x^{u}(t)$ is said to be a solution to (1) if $x^{u}(t)$ has the following properties

(i) $x^{u}(t)$ is $t$-continuous and $\mathcal{F}_{t}^{m}$-adapted;

(ii) $f(t, x(t), u(t, x(t))) \in \mathcal{L}^{1}\left([0, T] \times R^{n} ; U\right)$, $g(t, x(t), u(t, x(t))) \in \mathcal{L}^{2}\left([0, T] \times R^{n} ; U\right)$

(iii) $x^{u}(0)=\xi$, for each $t \in[0, T]$ and $u \in U$

$$
x^{u}(t)=\xi+\int_{0}^{t} f(s, x(s), u(s, x(s))) d s+\int_{0}^{t} g(s, x(s), u(s, x(s))) d B(s)
$$

The uniqueness means that if $x^{u}(t), y^{u}(t)$ are two $t$-continuous functions satisfying conditions (i)-(iii), then

$$
P\left\{\sup _{0 \leq t \leq T}\left|x^{u}(t)-y^{u}(t)\right|>0\right\}=0
$$

Now we establish the existence-and-uniqueness theorem for equation (1).

Theorem 3.2. Suppose that $g(t, x(t), u(t, x(t))$ and $f(t, x(t), u(t, x(t)))$ satisfy conditions $(i)-(v i)$, and $x^{u}(0)$ is a random variable which is independent of the $\sigma$-algebra $\mathcal{F}_{t}$ and $E\left|x^{u}(0)\right|^{2}<\infty$. Then there is a unique solution $x^{u}(t)$ to (1) defined on $[0, T], T \geq 0$.

Proof. First we prove the existence of solution. We define the Picard sequence

$$
\begin{aligned}
x_{n}^{u_{n}}(t)= & x_{0}^{u_{0}}(0)+\int_{0}^{t} f\left(s, x_{n-1}(s), u\left(s, x_{n-1}(s)\right)\right) d s \\
& +\int_{0}^{t} g\left(s, x_{n-1}(s), u\left(s, x_{n-1}(s)\right)\right) d B(s)
\end{aligned}
$$


here $u_{n}=u\left(s, x_{n}(s)\right)$. Now we prove that $x_{n}^{u_{n}}(t)$ is uniformly convergent in $[0, T]$. By the definition of $x_{n}^{u_{n}}(t)$, one can show that

$$
\begin{aligned}
& \left|x_{1}^{u_{1}}(t)-x_{0}^{u_{0}}(t)\right|^{2} \\
= & \left|\int_{0}^{t} f\left(s, x_{0}(s), u\left(s, x_{0}(s)\right)\right) d s+\int_{0}^{t} g\left(s, x_{0}(s), u\left(s, x_{0}(s)\right)\right) d B(s)\right|^{2}
\end{aligned}
$$

By the elementary inequality $(a+b)^{2} \leq 2\left(a^{2}+b^{2}\right)$, we get

$$
\begin{aligned}
& || x_{1}^{u_{1}}(t)-\left.x_{0}^{u_{0}}(t)\right|^{2} \\
\leq & 2\left|\int_{0}^{t} f\left(s, x_{0}(s), u\left(s, x_{0}(s)\right)\right) d s\right|^{2}+2\left|\int_{0}^{t} g\left(s, x_{0}(s), u\left(s, x_{0}(s)\right)\right) d B(s)\right|^{2}
\end{aligned}
$$

Taking the expectation on both sides, and by the following condition

$$
E\left|\int_{0}^{t} g\left(s, x_{0}(s), u\left(s, x_{0}(s)\right)\right) d B(s)\right|^{2}=\int_{0}^{t} E\left|g\left(s, x_{0}(s), u\left(s, x_{0}(s)\right)\right)\right|^{2} d s
$$

we obtain

$$
\begin{aligned}
& E\left|x_{1}^{u_{1}}(t)-x_{0}^{u_{0}}(t)\right|^{2} \\
\leq & 2 t \int_{0}^{t} E\left|f\left(s, x_{0}(s), u\left(s, x_{0}(s)\right)\right)\right|^{2} d s+2 \int_{0}^{t} E \mid g\left(s, x_{0}(s),\left.u\left(s, x_{0}(s)\right)\right|^{2} d s\right.
\end{aligned}
$$

Using (2) we get

$$
E\left|x_{1}^{u_{1}}(t)-x_{0}^{u_{0}}(t)\right|^{2} \leq 2 C T(T+1)\left(1+E\left|x_{0}(s)\right|^{2}+E\left|u\left(s, x_{0}(s)\right)\right|^{2}\right)
$$

Also using (4) we get

$$
E\left|x_{1}^{u_{1}}(t)-x_{0}^{u_{0}}(t)\right|^{2} \leq 2 C T(T+1)\left(1+E\left|x_{0}(s)\right|^{2}+M^{2}\right)
$$

So that

$$
E\left(\sup _{0 \leq s \leq t}\left|x_{1}^{u_{1}}(s)-x_{0}^{u_{0}}(s)\right|^{2}\right) \leq A_{1}
$$

where $A_{1}=2 C T(T+1)\left(1+E\left|x_{0}(s)\right|^{2}+M^{2}\right)$, We now claim that for all $n \geq 0$

$$
E\left(\sup _{0 \leq s \leq t}\left|x_{n+1}^{u_{n+1}}(s)-x_{n}^{u_{n}}(s)\right|^{2}\right) \leq \frac{A_{1}\left(A_{2} t\right)^{n}}{n \times(n-1) \times \cdots \times 1} \quad t \in[0, T]
$$

where $A_{2}$ only depends on $K, C, L, T, M$ and $E\left|x^{u}(0)\right|$. We shall show this by induction. In view of (6) we see that $(7)$ holds when $n=0$. Under the inductive assumption, (7) holds for some $n \geq 0$, so we shall show that (7) also holds for $n+1$. Note that

$$
\begin{aligned}
& E\left(\sup _{0 \leq s \leq t}\left|x_{n+2}^{u_{n+2}}(s)-x_{n+1}^{u_{n+1}}(s)\right|^{2}\right) \\
= & E\left(\sup _{0 \leq s \leq t} \mid \int_{0}^{t}\left[f\left(s, x_{n+1}(s), u\left(s, x_{n+1}(s)\right)\right)-f\left(s, x_{n}(s), u\left(s, x_{n}(s)\right)\right)\right] d s\right. \\
& \left.-\left.\int_{0}^{t}\left[g\left(s, x_{n+1}(s), u\left(s, x_{n+1}(s)\right)\right)-g\left(s, x_{n}(s), u\left(s, x_{n}(s)\right)\right)\right] d B(s)\right|^{2}\right)
\end{aligned}
$$


Taking the expectation, by (3) and (4) as well as the inductive assumption, we get

$$
\begin{aligned}
& E\left(\sup _{0 \leq s \leq t}\left|x_{n+2}^{u_{n+2}}(s)-x_{n+1}^{u_{n+1}}(s)\right|^{2}\right) \\
\leq & 2 K T(T+1)(L+1) \int_{0}^{t}\left|x_{n+1}^{u_{n+1}}(s)-x_{n}^{u_{n}}(s)\right|^{2} d s \\
\leq & A_{2} \int_{0}^{t} E\left(\sup _{0 \leq r \leq s}\left|x_{n+1}^{u_{n+1}}(r)-x_{n}^{u_{n}}(r)\right|^{2} d s\right. \\
\leq & A_{2} \int_{0}^{t} \frac{A_{1}\left(A_{2} s\right)^{n}}{n \times(n-1) \times \cdots \times 1} d s \\
= & \frac{A_{1}\left(A_{2} t\right)^{n+1}}{(n+1) \times n \times \cdots \times 1}
\end{aligned}
$$

That is (7) holds for $n+1$. So by induction, (7) holds for all $n \geq 0$. Thus,

$$
E\left(\sup _{0 \leq s \leq t}\left|x_{n+1}^{u_{n+1}}(s)-x_{n}^{u_{n}}(s)\right|^{2}\right) \leq \frac{A_{1}\left(A_{2} t\right)^{n}}{n \times(n-1) \times \cdots \times 1}
$$

By Lemma 2.1

$$
P\left\{\sup _{0 \leq s \leq t}\left|x_{n+1}^{u_{n+1}}(s)-x_{n}^{u_{n}}(s)\right| \geq \frac{1}{2^{n}}\right\} \leq \frac{A_{1}\left(4 A_{2} t\right)^{n}}{n \times(n-1) \times \cdots \times 1}
$$

Since $\sum_{n=0}^{\infty} \frac{A_{1}\left(4 A_{2} t\right)^{n}}{n \times(n-1) \times \cdots \times 1}<\infty$ So

$$
\sum_{n=0}^{\infty} P\left\{\sup _{0 \leq s \leq t}\left|x_{n+1}^{u_{n+1}}(s)-x_{n}^{u_{n}}(s)\right| \geq \frac{1}{2^{n}}\right\}<\infty
$$

By Lemma 2.2

$$
P\left\{\lim _{n \rightarrow \infty} \sup _{0 \leq s \leq t}\left|x_{n+1}^{u_{n+1}}(s)-x_{n}^{u_{n}}(s)\right| \geq \frac{1}{2^{n}}\right\}=0
$$

that is for every $u \in U$, there exists $n_{0} \in N$, when $n \geq n_{0}$, we have

$$
\sup _{0 \leq t \leq T}\left|x_{n+1}^{u_{n+1}}(t)-x_{n}^{u_{n}}(t)\right| \leq \frac{1}{2^{n}}
$$

Implies that

$$
\begin{aligned}
& a c-\lim _{n \rightarrow \infty}\left\{x_{0}^{u_{0}}(t)+\sum_{n=0}^{\infty}\left[x_{n+1}^{u_{n+1}}(t)-x_{n}^{u_{n}}(t)\right]\right\} \\
= & a c-\lim _{n \rightarrow \infty} x_{n}^{u_{n}}(t)=x^{u}(t)
\end{aligned}
$$

uniformly on $[0, T]$. Finally, we show that $x^{u}(t)$ satisfies (1).

Obviously, $x^{u}(t)$ is $t$-continuous and $\mathcal{F}_{t}^{m}$-adapted

Notice that

$$
E\left|\int_{0}^{t}\left[f\left(s, x_{n}(s), u\left(s, x_{n}(s)\right)\right)-f(s, x(s), u(s, x(s)))\right] d s\right|^{2}
$$




$$
\begin{aligned}
& +E\left|\int_{0}^{t}\left[g\left(s, x_{n}(s), u\left(s, x_{n}(s)\right)\right)-g(s, x(s), u(s, x(s)))\right] d B(s)\right|^{2} \\
\leq & K(T+1)(L+1) \int_{0}^{t} E\left|x_{n}^{u_{n}}(s)-x^{u}(s)\right|^{2} d s \rightarrow 0 \quad(n \rightarrow \infty)
\end{aligned}
$$

and

$$
\begin{aligned}
& a c-\lim _{n \rightarrow \infty} \int_{0}^{t} f\left(s, x_{n}(s), u\left(s, x_{n}(s)\right)\right) d s \\
= & \int_{0}^{t} f(s, x(s), u(s, x(s))) d s \quad t \in[0, T]
\end{aligned}
$$

By Lemma 2.3 we get

$$
\begin{aligned}
& s t-\lim _{n \rightarrow \infty} \int_{0}^{t} g\left(s, x_{n}(s), u\left(s, x_{n}(s)\right)\right) d B(s) \\
= & \int_{0}^{t} g(s, x(s), u(s, x(s))) d B(s) \quad t \in[0, T]
\end{aligned}
$$

Therefore taking limit of

$$
\begin{aligned}
x_{n}^{u_{n}}(t)= & x_{0}^{u_{0}}(0)+\int_{0}^{t} f\left(s, x_{n-1}(s), u\left(s, x_{n-1}(s)\right)\right) d s \\
& +\int_{0}^{t} g\left(s, x_{n-1}(s), u\left(s, x_{n-1}(s)\right)\right) d B(s)
\end{aligned}
$$

as $n \rightarrow \infty$, we have

$$
\begin{aligned}
x^{u}(t)= & x_{0}^{u_{0}}(0)+\int_{0}^{t} f(s, x(s), u(s, x(s))) d s \\
& +\int_{0}^{t} g(s, x(s), u(s, x(s))) d B(s)
\end{aligned}
$$

So $x^{u}(t)$ is the solution of $(1)$.

Next we give the proof of the uniqueness. Let $x^{u}(t)$ and $y^{u}(t)$ be solution to (1), then

$$
\begin{aligned}
\left|x^{u}(t)-y^{u}(t)\right|^{2}= & \mid \int_{0}^{t}[f(s, x(s), u(s, x(s)))-f(s, y(s), u(s, y(s)))] d s \\
& +\left.\int_{0}^{t}[g(s, x(s), u(s, x(s)))-g(s, y(s), u(s, y(s)))] d B(s)\right|^{2}
\end{aligned}
$$

By the elementary inequality $(a+b)^{2} \leq 2\left(a^{2}+b^{2}\right)$ we get

$$
\begin{aligned}
& E\left|x^{u}(t)-y^{u}(t)\right|^{2} \\
\leq & 2 K(T+1)(L+1) \int_{0}^{t} E\left|x^{u}(s)-y^{u}(s)\right|^{2} d s
\end{aligned}
$$


By Lemma 2.4 we conclude that

$$
E\left(\sup _{0 \leq t \leq T}\left|x^{u}(s)-y^{u}(s)\right|^{2}\right)=0
$$

that is

$$
\int_{\Omega} \sup _{0 \leq s \leq t}\left|x^{u}(s)-y^{u}(s)\right|^{2} d p=0 \quad t \in[0, T]
$$

So

$$
\sup _{0 \leq t \leq T}\left|x^{u}(s)-y^{u}(s)\right|=0 \quad \text { a.s. } \quad \text { for all } t \in[0, T], u \in U .
$$

That is

$$
P\left\{\sup _{0 \leq t \leq T}\left|x^{u}(t)-y^{u}(t)\right|>0\right\}=0
$$

Theorem 3.3. Under the conditions of Theorem 3.2, the solution of (1) satisfies

$$
\sup _{0 \leq t \leq T} E\left|x^{u}(t)\right|^{2} \leq 3\left\{E|\xi|^{2}+C T(T+1)(M+1)\right\} e^{3 C T(T+1)}
$$

Proof. Let

$$
x^{u}(t)=\xi+\int_{0}^{t} f(s, x(s), u(s, x(s))) d s+\int_{0}^{t} g(s, x(s), u(s, x(s))) d B(s)
$$

is the solution of (1).

By the elementary inequality $(a+b+c)^{2} \leq 3\left(a^{2}+b^{2}+c^{2}\right)$, we get

$$
\begin{aligned}
\left|x^{u}(t)\right|^{2} \leq & 3\left\{|\xi|^{2}+\left|\int_{0}^{t} f(s, x(s), u(s, x(s))) d s\right|^{2}\right. \\
& \left.+\left|\int_{0}^{t} g(s, x(s), u(s, x(s))) d B(s)\right|^{2}\right\}
\end{aligned}
$$

Taking the expectation on both sides, by Cauchy-Schwarz inequality and condition (2) we obtain

$$
\begin{aligned}
& E\left|x^{u}(t)\right|^{2} \\
\leq & 3\left\{E|\xi|^{2}+T \int_{0}^{t} E|f(s, x(s), u(s, x(s)))|^{2} d s\right. \\
& \left.+\int_{0}^{t} E|g(s, x(s), u(s, x(s)))|^{2} d s\right\} \\
\leq & 3\left\{E|\xi|^{2}+C(T+1)\left(\int_{0}^{t}\left(1+E\left|x^{u}(s)\right|^{2}\right) d s+T M\right)\right\} \\
= & 3 E|\xi|^{2}+3 C(T+1)\left(\int_{0}^{t} E\left|x^{u}(s)\right|^{2} d s+T M+T\right)
\end{aligned}
$$


For any $t_{1} \in[0, T]$.

$$
\begin{aligned}
& \sup _{0 \leq t \leq t_{1}} E\left|x^{u}(t)\right|^{2} \\
\leq & 3 E|\xi|^{2}+3 C T(T+1)(M+1)+3 C(T+1) \int_{0}^{t} \sup _{0 \leq S \leq t} E\left|x^{u}(s)\right|^{2} d s
\end{aligned}
$$

So, by Lemma 2.4 we have

$$
\begin{aligned}
& \sup _{0 \leq t \leq T} E\left|x^{u}(t)\right|^{2} \\
\leq & 3\left[E|\xi|^{2}+C T(T+1)(M+1)\right] e^{3 C T(T+1)}
\end{aligned}
$$

which is the required assertion (8).

\section{Conclusion}

For stochastic control system (1), applying the mean square linear growth condition and mean square Lipschitz condition, we give and prove the existence and uniqueness theorem, and then investigate the behavior of the solutions. The main results are given in Theorem 3.2 and Theorem 3.3 in Section 3. It is shown that if the set of control functions is a bounded set, and control function satisfies mean square Lipschitz condition, the stochastic control system (1) has a unique solution.

\section{REFERENCES}

1. M. Chang, Stochatic Control of Hereditary Systems and Applications, Springer, New York, 2008.

2. M. Arriojas, Y. Hu, S. Monhammed and G. Pap, A delayed Black and Scholes formula, Stochastic Analysis and Applications, (2007), 471-492.

3. Bernt $\varnothing$ ksendal, Stochastic Differential Equations, 6th Edition, Springer-Verlag, Berlin, 2006.

4. G. Hu, K. Wang, Existence and uniqueness of solutions to stochastic differential equation with random coefficients, Ann. of Diff. Eqs. 26, (2010), 400-406.

5. Arnold Ludwig, Stochastic Differential Equations: Theory and Applications, Montreal, Quebec, 1971.

6. L. Chen, Z. Wu, A type of general forward-backward stochastic differential equations and applications, Chinese Annals of Mathematics, 32B(2), (2011), 279-292.

7. Q. Zhu, Y. Shi, Backward doubly stochastic differential equations with jumps and stochastic partial differential-integral equations, Chinese Annals of Mathematics, 33B(1), (2012), 127142 .

Peilin Lu received B.Sc. from Baotou Teachers College. She is now a master student in Inner Mongolia University. Her research interests include stochastic control systems and viability theory.

School of Mathematical Sciences, Inner Mongolia University, 235 West Daxue Road, Hohhot 010021, China.

e-mail: lupeilin85@163.com 
Caixia Gao received M.Sc. from Inner Mongolia University, and Ph.D. from Dalian University of Technology. She is currently an associate professor at Inner Mongolia University since 2006. Her research interests are optimization, control theory and their applications.

School of Mathematical Sciences, Inner Mongolia University, 235 West Daxue Road, Hohhot 010021, China.

e-mail: gaocx0471@163.com 